\title{
Recent Advances in the Diagnosis and Treatment of Gastrointestinal Diseases
}

The 10th Annual Meeting of the Japanese Gastroenterological Association (JGA) was organized by Prof. Seiichi Takenoshita and held in Fukushima on February 14 and 15,2013. During the meeting, a 10th anniversary review session was held in addition to the regular program. This session focused on recent advances in the diagnosis and treatment of gastrointestinal diseases, particularly gastrointestinal cancers.

The articles published in the upcoming (2015) JGA Special Issue of Digestion represent the best presentations from the 10th Annual Meeting. The session chairpersons recommended numerous papers from their sections. The editorial committee of the JGA subsequently selected distinguished presentations from those recommendations and invited the authors to submit their papers for this issue. Two reviews and 10 original articles were accepted for final publication. All manuscripts were independently reviewed by two members of the JGA editorial committee.

Advances in the diagnosis and treatment of gastrointestinal cancers have been remarkable in the recent years. In this issue, one review focuses on new diagnostic procedures and novel therapeutic strategies for gastrointestinal stromal tumors and neuroendocrine tumors. Gastric cancer is the second leading cause of cancer death in Japan. One article describes the relationship between the degree of endoscopic atrophy of the gastric mucosa and carcinogenic risk. The serrated neoplasia pathway has attracted attention as an alternative pathway involved in colorectal cancer development. Another article describes the clinicopathological and molecular characteristics of serrated lesions in elderly Japanese patients. In Japan, endoscopic submucosal dissection (ESD) has been widely accepted as a reliable procedure that enables en bloc resection of large superficial lesions in the upper gastrointestinal tract. An article here describes the utility of ESD in total excisional biopsy for clinical $\mathrm{T} 1$ colorectal cancer. The usefulness of a new device, named Smart Shooter, for endoscopic treatment is described in another article. A cell surface-associated enzyme, $\gamma$-glutamyl-transpeptidase (GGT), is overexpressed in colorectal cancers. One of the articles describes a pilot study of ex vivo fluorescent imaging of colorectal tumors using a GGT-activatable fluorescent probe.

In addition, this issue contains a review and an original article describing the treatment of inflammatory bowel disease. The original article describes a novel therapeutic strategy for Crohn's disease patients with a loss of response to infliximab. The review describes the therapeutic potential of carbon monoxide in inflammatory bowel disease. Capsule endoscopy is widely used to evaluate suspected small bowel disease. However, some patients with 
small bowel disease, including Crohn's disease, are at risk of capsule retention. An article included here describes the efficacy and safety of a patency capsule to assess the patency of the small bowel prior to capsule endoscopy.

Helicobacter pylori infection and nonsteroidal anti-inflammatory drugs (NSAIDs) are the two main causes of peptic ulcer bleeding. An article in this issue describes the shift from $H$. pylori infection to the use of NSAIDs as the main cause of peptic ulcer bleeding in Japan. The treatment of proton pump inhibitor (PPI)-resistant nonerosive reflux disease (NERD) is an important issue in the management of gastroesophageal reflux disease. Another article describes the usefulness of esophageal manometry in diagnosing PPI-resistant NERD.
Acute pancreatitis is a serious complication associated with endoscopic retrograde cholangiopancreatography (ERCP). Papillary edema has been implicated as a cause of post-ERCP pancreatitis. In this issue, an article describes the preventive effect of Shakuyakukanzoto (TJ68), a Kampo medicine, on post-ERCP pancreatitis.

We believe this issue will provide useful information and lead to further advances in the diagnosis and treatment of gastrointestinal diseases. We wish to express our sincere gratitude to all the authors and chairpersons for their contributions to this special issue.

Yasuhisa Shinomura, MD, PhD

Chairperson of the

Associated Journal Committee of the JGA 